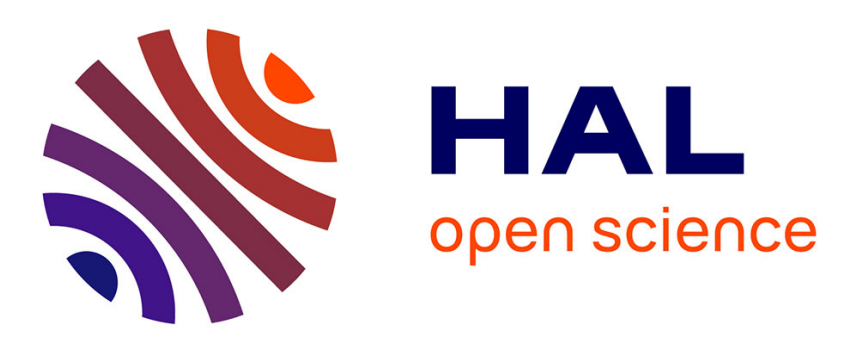

\title{
Fibroblasts Control Macrophage Differentiation during Pulp Inflammation
}

\author{
Chloé Le Fournis, Charlotte Jeanneau, Thomas Giraud, Ikhlas El Karim, \\ Fionnuala Lundy, Imad About
}

\section{- To cite this version:}

Chloé Le Fournis, Charlotte Jeanneau, Thomas Giraud, Ikhlas El Karim, Fionnuala Lundy, et al.. Fibroblasts Control Macrophage Differentiation during Pulp Inflammation. Journal of Endodontics, 2021, 47 (9), pp.1427-1434. 10.1016/j.joen.2021.06.015 . hal-03547528

\section{HAL Id: hal-03547528 \\ https://hal.science/hal-03547528}

Submitted on 28 Jan 2022

HAL is a multi-disciplinary open access archive for the deposit and dissemination of scientific research documents, whether they are published or not. The documents may come from teaching and research institutions in France or abroad, or from public or private research centers.
L'archive ouverte pluridisciplinaire HAL, est destinée au dépôt et à la diffusion de documents scientifiques de niveau recherche, publiés ou non, émanant des établissements d'enseignement et de recherche français ou étrangers, des laboratoires publics ou privés. 


\section{Macrophage Differentiation during Pulp Inflammation}

\begin{abstract}
Introduction: During pulp inflammation, recruited macrophages can differentiate into 2 phenotypes: proinflammatory M1 and anti-inflammatory M2. Pulp fibroblasts have previously been shown to regulate pulp inflammation via cytokine and growth factor secretion. We hypothesized that upon carious injury, pulp fibroblasts interact with macrophages and modulate their differentiation. Methods: Cultures of pulp fibroblasts were physically injured and incubated with lipoteichoic acid (LTA) to mimic the pulp environment underlying a carious lesion. Physical injuries without LTA were performed on cultured fibroblasts to simulate the surrounding pulp tissue. Fibroblast supernatants were collected and added to undifferentiated macrophages to study their differentiation into M1 or M2 phenotypes by investigating cytokine secretion profiles and phagocytosis capacity. Histologic staining and immunofluorescence were performed on healthy and carious human tooth sections to localize the 2 macrophage phenotypes. Results: LTA-stimulated fibroblasts induced macrophage differentiation into the M1 phenotype with a significant increase both in tumor necrosis factor alpha secretion and phagocytosis capacity. By contrast, injured fibroblasts without LTA led to M2 differentiation with a significant increase in interleukin 10 secretion and low phagocytosis capacity. In carious teeth, M1 macrophages were detected mainly in the pulp zone underlying caries, whereas M2 macrophages were detected in the peripheral inflammatory zone.

Conclusions: Fibroblasts induced macrophage differentiation to proinflammatory M1 with high bacteria phagocytosis capacity to control infection at the carious front. Fibroblasts located at the periphery of the inflammatory zone induced macrophage differentiation to antiinflammatory M2. The fine balance between the 2 phenotypes may represent a prerequisite for initiating the healing process. (J Endod 2021;47:1427-1434.)
\end{abstract}

\section{KEY WORDS}

Caries; macrophage differentiation; pulp biology; pulp fibroblast; pulp inflammation control

When cariogenic bacteria infiltrate the dentin-pulp tissue during the carious process, resident cells such as odontoblasts, fibroblasts, macrophages, mast cells, and dendritic cells produce signals to initiate inflammation ${ }^{1-3}$. White blood cells such as neutrophils and monocytes are recruited to the infected pulp zone, and this immune cell migration is guided by chemokines such as interleukin (IL)- 8 secreted by odontoblasts or Complement C5a by fibroblasts ${ }^{4,5}$. Circulating monocytes migrate toward the infected pulp tissue and are activated/differentiated into macrophages.

Macrophages represent the major pulp immunocompetent cells in fighting cariogenic bacteria ${ }^{6,7}$. Depending on their environment, macrophages can adopt 2 phenotypes: M1 and M2. M1 differentiation occurs in response to intracellular pathogens, bacterial lipopolysaccharides (LPSs), and cytokines such as interferon gamma (IFN- $\gamma$ ) and tumor necrosis factor alpha (TNF- $\alpha$ ) secreted by Th1/natural killer cells ${ }^{8}$. M1 macrophages mediate the host defense against pathogens secreting proinflammatory cytokines (IL$1 \beta$, TNF- $\alpha$, and IL-6), reactive oxygen species, nitric oxide, and phagocyte foreign materials ${ }^{9}$. By contrast, M2 macrophages are activated by cytokines secreted by Th2/mast cells, such as IL-4, IL-13, IL-10, or transforming growth factor beta $(\text { TGF- } \beta)^{8}$. M2 macrophages contribute to tissue repair by producing antiinflammatory cytokines such as IL-10, TGF- $\beta$, and vascular endothelial growth factor ${ }^{10}$. Thus, M1 and M2 macrophages have the potential to contribute to pulpal inflammation and healing processes. Previous studies reported that TNF- $\alpha, I L-1 \beta, C X C$ chemokine ligand 10 , and macrophage inflammatory protein $3 a$ cytokines were detected in inflamed pulp, suggesting a predominance of M1 macrophages in response
Chloé Le Fournis, PhD, *

Charlotte Jeanneau, PhD, *

Thomas Giraud, PhD, ${ }^{*+}$

Ikhlas El Karim, PhD, ${ }^{\neq}$

Fionnuala T. Lundy, PhD, ${ }^{\ddagger}$ and Imad About, $P h D^{*}$

\section{SIGNIFICANCE}

Depending on the stimulation type, pulp fibroblasts induce macrophage differentiation into proinflammatory $\mathrm{M} 1$ or antiinflammatory M2 occupying distinct locations within the pulp inflammation zone. This differentiation is essential for bacteria elimination and pulp protection during the carious process. 
to caries progression ${ }^{11-13}$. M2 macrophages have been associated with dentin bridge formation; indeed, TGF- $\beta$ secreted by M2 macrophages increase the alkaline phosphatase activity of dental pulp cells involved in new dentin synthesis after pulp exposure $^{14}$. Similarly, a transient M2 accumulation is correlated with a thin layer of dentin formation in contact with mineral trioxide aggregate after pulpotomy ${ }^{15}$. Recent work showed colocalization of M2 macrophages with Schwann cells in healthy dental pulp, under caries, and during the wound healing/mineralized bridge formation after pulp capping with mineral trioxide aggregate $^{16}$. Therefore, M1 macrophages regulate the inflammatory reaction in the dental pulp, whereas M2 macrophages appear to be involved in wound healing. M1 and M2 polarization is governed mainly by immune cell secretion, but recently nonimmune cells such as cardiac fibroblasts were shown to play a significant role in modulating macrophage polarization ${ }^{17}$. Indeed, LPS-stimulated cardiac fibroblasts promoted the macrophage M1 phenotype, whereas TGF- $\beta 1$ stimulation of these cells induced the M2 phenotype ${ }^{17}$. This macrophage polarization is essential for cardiac remodeling after injury, evolving from an initial accumulation of proinflammatory M1 macrophages to a greater balance of antiinflammatory M2 macrophages. Dental pulp fibroblasts have unique properties, and recent data have shown that these cells play important functions in controlling pulp vascularization, infection, and regeneration ${ }^{18-}$ ${ }^{23}$. Thus, although cardiac fibroblast stimulation affects macrophage polarization and the subsequent inflammation/healing process, the effect of fibroblasts on macrophage differentiation has never been investigated in pulp tissue.

This work aimed to study the role of pulp fibroblasts on macrophage differentiation during the carious process. We investigated the location of M1 and M2 macrophages in healthy and carious tooth sections and hypothesized that during the carious process pulp fibroblasts could be subjected to 2 types of stimulation depending on their location. Those located directly beneath the carious site could be exposed to cariogenic bacteria or macromolecules such as lipoteichoic acid (LTA), whereas fibroblasts located at the periphery of the inflammatory zone may be modulated by the inflammatory process without any contact with bacteria or their components. Cells in this zone receive signals from the surrounding environment because of the inflammatory reaction including damageassociated molecular pattern molecules
(DAMPs) and immunostimulatory molecules of tissue repair ${ }^{24,25}$.

To simulate the fibroblast environment beneath the carious site, fibroblasts were physically injured and incubated with LTA while the cell environment at the periphery of the inflammatory zone was simulated by physical injury to fibroblasts (without LTA). Fibroblast supernatants were then collected and incubated with undifferentiated macrophages. The subsequent macrophage differentiation was studied by investigating proinflammatory TNF- $\alpha$ and anti-inflammatory IL-10 cytokine secretion, measuring their bacterial phagocytic capacity and comparing them with differentiated $\mathrm{M} 1$ and $\mathrm{M} 2$ as reference phenotypes.

\section{MATERIALS AND METHODS}

\section{Reagents}

Cell culture reagents were purchased from Dominique Dutscher (Brumath, France), primary antibodies from R\&D Systems (Lille, France) and Alexa Fluor secondary antibodies from Life Technologies (Saint-Aubin, France).

\section{Teeth}

Immature human third molars freshly extracted for orthodontics reasons and carious teeth were obtained in compliance with French legislation (informed patient consent and institutional review board approval of the protocol used).

\section{Bacterial Culture}

Streptococcus mutans (American Type Culture Collection 31383) was obtained from American Type Culture Collection (Manassas, VA) and cultured at $37^{\circ} \mathrm{C}$ under aerobic condition in brain-heart infusion broth (Conda) Pronadisa, Madrid, Spain).

\section{Pulp Fibroblast Cell Culture and Treatment}

Primary pulp cells were prepared from immature third molars by the explant outgrowth method. Pulp fibroblasts were isolated from pulp cell cultures and characterized as previously described ${ }^{18,26}$. These cells were cultured in minimal essential medium (MEM) supplemented with 10\% fetal bovine serum, L-glutamine $2 \mathrm{mmol} / \mathrm{L}$, penicillin/streptomycin $50 \mu \mathrm{g} / \mathrm{mL}$, and amphotericin $B 0.25 \mu \mathrm{g} / \mathrm{mL}$ at $37^{\circ} \mathrm{C}$ in a $5 \%$ $\mathrm{CO}_{2}$ atmosphere.

To simulate the zone under the carious lesion, confluent pulp fibroblasts were incubated with $1 \mu \mathrm{g} / \mathrm{mL}$ LTA (InvivoGen, San Diego, CA) for 4 hours. Then, physical injuries were performed with sterile scalpels in vertical and horizontal directions (10 in each direction in 6-well plates) in serum-free MEM. To simulate the cell environment at a distance from the caries front, confluent fibroblasts were physically injured and incubated in serum-free MEM. The fibroblast supernatants were harvested after 24 hours and used for subsequent macrophage differentiation experiments as "LTA + injury" or "injury." Fibroblast cultures without physical injury or LTA were used as controls and referred to as "untreated."

\section{THP-1 Cell Culture}

THP-1 cells, a human monocytic cell line (Sigma-Aldrich, St Louis, MO), were cultured in Roswell Park Memorial Institute (RPMI) medium as described previously ${ }^{27}$.

\section{Macrophage Differentiation}

Undifferentiated MO macrophages were obtained by incubating THP-1 cells with 100 ng/mL phorbol 12-myristate 13-acetate (PMA, InvivoGen) for 48 hours. M1 macrophages were obtained by incubating $M 0$ in serum-free RPMI with a combination of PMA (100 ng/mL) and LPS (100 ng/mL, R\&D Systems)/IFN- $\gamma$ (20 ng/mL, R\&D Systems) for 24 hours. M2 macrophages were obtained by incubating $\mathrm{MO}$ in serum-free RPMI with a combination of PMA (100 ng/mL) and IL-4 (20 ng/mL, R\&D Systems) for 48 hours. This differentiation protocol was established based on recommendations by Murray et $\mathrm{al}^{28}$. To study fibroblast impact on macrophage differentiation, M0 macrophages were incubated for 48 hours with stimulated fibroblast supernatants containing PMA (100 ng/mL). M0 macrophages were incubated with injured fibroblast supernatant "injury" or LTA and injured fibroblast supernatant "LTA + injury." The macrophage differentiation protocol is summarized in Figure 1.

\section{Cytokine Secretion by Macrophages}

After MO macrophage incubation with fibroblast supernatants, cells were washed 3 times with phosphate-buffered saline. Serumfree RPMI (2 $\mathrm{mL}$ in 6-well plate) was added to macrophages for 24 hours. TNF- $\alpha$ and IL-10 were quantified in macrophage supernatants by enzyme-linked immunosorbent assay in 96well plates (Nunc Maxisorp, Dominique Dutscher) using the Duoset human TNF- $\alpha$ or IL-10 kits (R\&D Systems) according to the manufacturer's instructions. All experiments were performed in triplicate. 


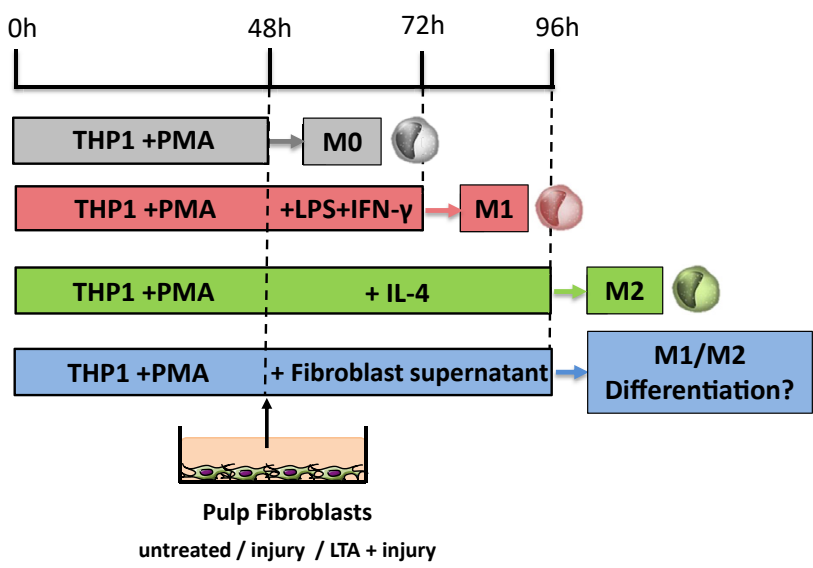

FIGURE 1 - The macrophage differentiation protocol. Undifferentiated M0 macrophages were obtained by incubating THP-1 monocytes with PMA (100 ng/mL). M0 macrophages were incubated with PMA (100 ng/mL)/LPS (100 ng/mL)/ IFN- $\gamma(20 \mathrm{ng} / \mathrm{mL})$ for 24 hours to obtain M1 or PMA (100 ng/mL)/L-4 (20 ng/mL) for 48 hours to obtain M2 differentiation. The impact of pulp fibroblasts on macrophage differentiation was studied by incubating M0 macrophages with a combination of PMA (100 ng/mL) and fibroblast supernatants after different stimulation conditions for 48 hours. To quantify cytokine secretion by macrophages, the fibroblast supernatants incubated with M0 macrophages were discarded. Macrophages were washed 3 times with phosphate-buffered saline, and then they were incubated with a fresh serum-free medium for cytokine quantification.

\section{Quantification of Cariogenic Bacteria Phagocytosis by Macrophages}

Bacterial ingestion by macrophages was measured using a gentamicin protection assay $^{29}$. S. mutans suspension $\left(10^{7}\right.$ bacteria/ well) in serum-free RPMl was added to macrophages differentiated under the previously described conditions for 2 hours. Extracellular bacteria were killed with $200 \mu \mathrm{g} /$ $\mathrm{mL}$ gentamicin for 1 hour at $37^{\circ} \mathrm{C}$, and intracellular bacteria were released by cell lysis using $0.1 \%$ Triton X-100 (Sigma-Aldrich) for 5 minutes at room temperature. Intracellular bacteria were then diluted, and $100 \mu \mathrm{L}$ was spread on the agar plate (brain-heart infusion agar, Conda/Pronadisa) in triplicate. After incubation for 24 hours at $37^{\circ} \mathrm{C}$, colonyforming units (CFUs) were counted, and the average was calculated. Results were expressed in CFU/mL using the following equation:

$$
\begin{aligned}
\mathrm{CFU} / \mathrm{ml} & =\frac{\text { number of counted colonies }}{\text { volume of spread suspension }} \\
& \times \text { Dilution factor }
\end{aligned}
$$

Negative controls were performed by incubating macrophages in each condition with Cytochalasin D (Sigma-Aldrich) at a nontoxic concentration (10 $\mu \mathrm{mol} / \mathrm{mL})$.

\footnotetext{
Histology and Immunofluorescence Carious and intact teeth were fixed and routinely processed to obtain tooth sections as described previously ${ }^{30}$. After rehydration,
}

nonspecific binding sites were blocked with phosphate-buffered saline/3\% bovine serum albumin for 1 hour at room temperature.

Sections were then incubated overnight at $4^{\circ} \mathrm{C}$ with mouse immunoglobulin (lg) $\mathrm{G}$ antihuman CD86 $(5 \mu \mathrm{g} / \mathrm{mL})$ or mouse lgG antihuman CD206 $(5 \mu \mathrm{g} / \mathrm{mL})$ followed by secondary antibody Alexa Fluor 488 goat antimouse lgG ( $2 \mu \mathrm{g} / \mathrm{mL}$ ) or Alexa Fluor 594 donkey antimouse $\operatorname{lgG}(2 \mu \mathrm{g} / \mathrm{mL})$, respectively, for 45 minutes. Isotype controls were performed with control lgG followed by the secondary antibodies. Nuclei were counterstained with $1 \mu \mathrm{g} / \mathrm{mL}$ 4',6-diamidino-2-phenylindole (DAPI) for 45 minutes. Some sections were stained with hematoxylin-eosin (H\&E, Sigma-Aldrich) according to the manufacturer's instructions.

\section{Statistical Analysis}

All experiments were repeated at least 3 times, and statistical significance $(P<.05)$ was determined after checking normal distribution using the Student $t$ test to compare the different incubation conditions. Data are expressed as means \pm standard deviation.

\section{RESULTS}

\section{Fibroblasts Affect TNF- $\alpha$ and IL-10 Secretion by Macrophages} Undifferentiated M0 macrophages secreted a moderate level of TNF- $\alpha$ but did not secrete IL10. M1 macrophages secreted a higher TNF- $\alpha$ level than $\mathrm{MO}$ but did not secrete the antiinflammatory IL-10 (Fig. 2A and B). M2 macrophages did not secrete TNF- $\alpha$ but secreted a higher level of IL-10 than MO. When MO macrophages were incubated with untreated fibroblast supernatants, no change in TNF- $\alpha$ or IL-10 levels was observed. After incubation of $\mathrm{MO}$ with injured fibroblast supernatants, the TNF- $\alpha$ secretion level was lower than that of $\mathrm{MO}$, whereas the level of IL10 was comparable with that of M2. Incubation of $\mathrm{MO}$ with injured and LTA-stimulated fibroblast supernatants led to a significant increase in TNF- $\alpha$ levels equivalent to M1, whereas IL-10 levels were significantly lower than M2 (Fig. $2 A$ and $B$ ).

\section{Pulp Fibroblasts Affect Macrophage Phagocytosis Capacity}

Undifferentiated (M0) as well as differentiated M1 and M2 macrophages phagocytosed $S$. mutans (Fig. 2C). However, phagocytosis by $\mathrm{M} 1$ and $\mathrm{M} 2$ was significantly higher than that of M0. Moreover, M1 phagocytosis capacity was 40 times higher than M2. When M0 macrophages were incubated with untreated fibroblast supernatants, their phagocytic capacity did not change. In contrast, MO incubated with injured fibroblast supernatants had comparable phagocytosis capacity with M2 with no statistically significant difference (Fig. 2C). Moreover, when MO macrophages were incubated with injured and LTA-induced fibroblast supernatants, their phagocytosis capacity was comparable with $\mathrm{M} 1$. In the presence of cytochalasin $D$, an inhibitor of F-actin-dependent phagocytosis, a drastic decrease of CFUs was observed, indicating that the counted bacteria were engulfed by phagocytosis and present intracellularly (Fig. 2C).

\section{M1 Macrophages Are Mainly Located at the Central Inflammatory Area, Whereas M2 Macrophages Are Mostly Located in the Underlying Tissue at the Periphery of the Inflammatory Area} $\mathrm{H} \& E$ staining (Fig. $3 A$ and $B$ ) on healthy tooth sections showed a well-organized pulp tissue. No expression of CD86 or CD206 was observed by immunofluorescence (Fig. $3 \mathrm{C}$ and $D$ ), indicating that M1 and M2 macrophages were not detected. H\&E-stained carious tooth sections showed partial dentin destruction (black arrow, Fig. 3F) by cariogenic bacteria and pulp inflammation (limited by red dotted line, Fig. 3K). The expression of CD86 (red) and CD206 (green) was investigated in 2 areas: the center (Fig. 3G, magenta frame) and the periphery of the inflammation zone (Fig. $3 L$, green frame). The majority of M1 macrophages expressing CD86 were observed within the 

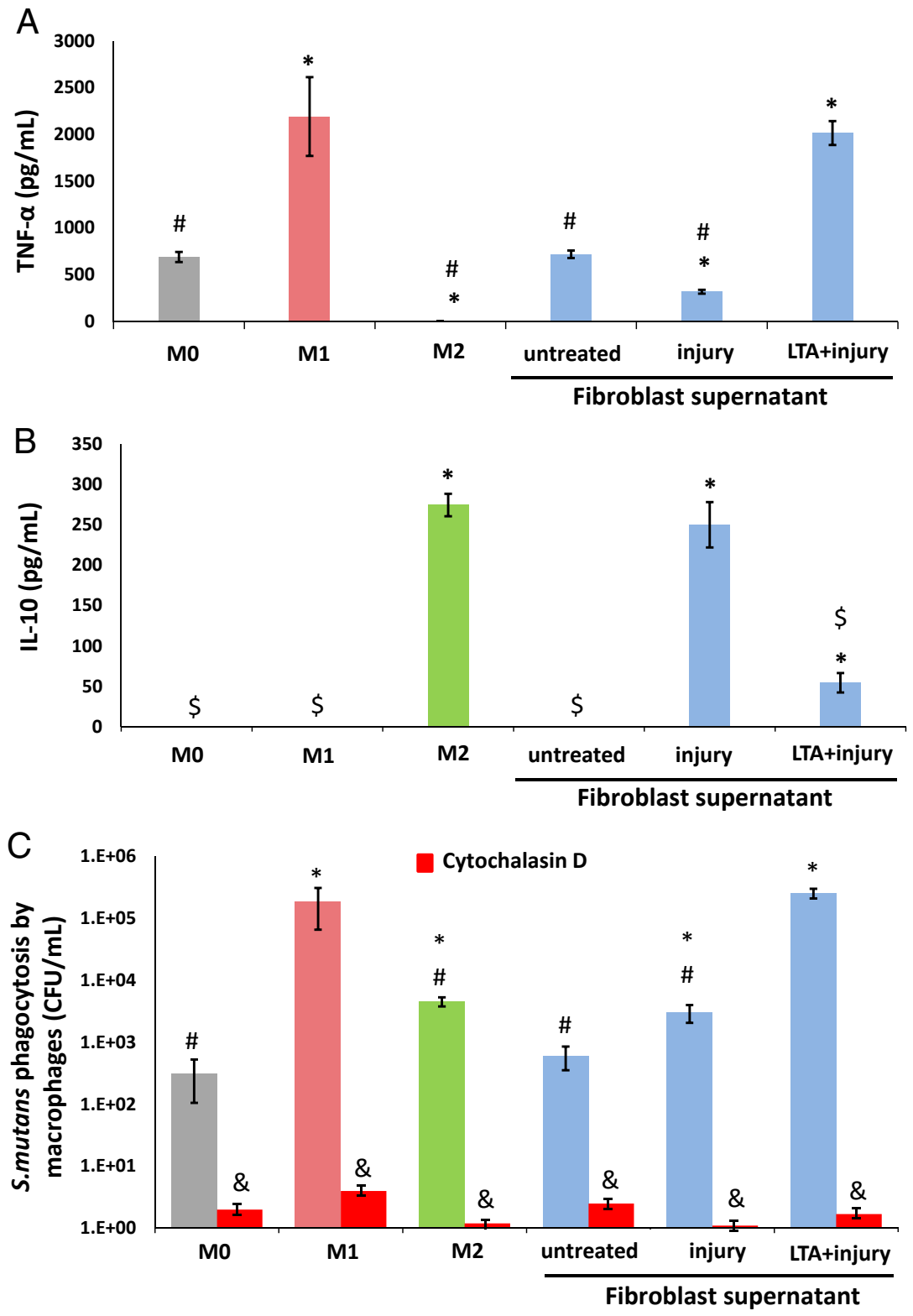

FIGURE 2 - The effect of fibroblasts on macrophage cytokine secretion and phagocytosis capacity. (A) Proinflammatory TNF- $\alpha$ cytokine. Undifferentiated MO macrophages and MO macrophages incubated with untreated fibroblast supernatants secrete moderate levels of TNF- $\alpha$. M1 macrophages and macrophages incubated with injured and LTAinduced fibroblast supernatants secrete a high level of TNF- $\alpha$. A low level of TNF- $\alpha$ is observed with injured fibroblast supernatants, whereas no TNF- $\alpha$ was secreted by M2 macrophages. (B) Anti-inflammatory IL-10 cytokine. M2 macrophages and MO macrophages incubated with injured fibroblast supernatants secrete a high level of IL-10 levels with no significant difference between the 2 conditions. Undifferentiated M0 macrophages, M0 macrophages incubated with untreated fibroblast supernatants, and M1 macrophages do not secrete IL-10. M0 macrophages incubated with injured and LTA-induced fibroblast secrete a low level of IL-10. (C) Macrophage phagocytosis capacity. Phagocytosis of S. mutans by M1 macrophages and M0 macrophages incubated with injured and LTA-induced fibroblast supernatants showed the highest phagocytosis capacity compared with the M0 control. Phagocytosis of $S$. mutans by M2 macrophages and M0 macrophages incubated with injured fibroblast supernatants was high but 40 times less than M1 macrophages. When M0 macrophages were incubated with untreated fibroblast supernatants, their phagocytic capacity did not change. A very low number of viable intracellular bacteria was observed when macrophages were treated with cytochalasin $D$, indicating a drastic inhibition of phagocytosis. The results are represented in a logarithmic scale. ${ }^{*} \mathrm{~A}$ statistical difference when a condition is compared with $\mathrm{M} 0(P<.05)$. \#A statistical difference when a condition is compared with M1 $(P<.05)$. \$A statistical difference when a condition is compared with M2 $(P<.05)$. \&A statistical difference when a condition is compared with cytochalasin $D$ treatment $(P<.05)$. Bars represent mean values \pm standard deviation. inflammatory area (Fig. $3 H$ and $M$ ), whereas M2 macrophages expressing CD206 were mainly detected at the periphery of the inflammatory zone (Fig. $3 /$ and $N$ ). No labeling was observed with the control isotypes (Fig. 3E, J, and O).

\section{DISCUSSION}

The main outcome of this study is that, depending on their stimulation conditions, pulp fibroblasts induce macrophage differentiation into proinflammatory $\mathrm{M} 1$ or anti-inflammatory M2 macrophages that occupy distinct locations within the inflammation zone. This macrophage differentiation is essential for cariogenic bacteria elimination as well as for pulp tissue protection from the extension of the inflammation during the carious process.

For this study, undifferentiated (MO) and differentiated (M1 and M2) macrophages were used as reference phenotypes to investigate the effect of fibroblast stimulation on macrophage cytokine secretion and phagocytic activity; MO macrophages were incubated with differentiation media to obtain M1 and M2 populations. The characterization of M1 and M2 in our study was based on surface markers, cytokine secretion, and phagocytic capacity. Although M1 and M2 express several surface markers, $M 1$ macrophages have been shown to strongly express CD86 and secrete a high level of TNF$\alpha$ and IL-12, whereas M2 macrophages have been shown to strongly express CD206 and secrete a high level of IL-10 and TGF- $\beta 1^{31}$. Although a wide range of surface markers and cytokines can be used, we selected CD86/ TNF- $\alpha$ to identify M1 and IL-10/CD206 to identify $\mathrm{M} 2$ because these are the most widely used in the characterization of the 2 phenotypes $^{31-33}$. Thus, studying TNF- $\alpha$ and IL-10 secretion in our work allows us to associate CD86 expression with M1 differentiation and CD206 expression with M2 differentiation.

In agreement with previously published data $^{8}$, our results show that $\mathrm{M} 1$ macrophages secrete high TNF- $\alpha$ levels and have high phagocytosis capacity, whereas M2 macrophages secrete high IL-10 levels and have moderate phagocytosis capacity. These profiles are clearly different from undifferentiated macrophages that secrete low TNF- $\alpha$ levels and no IL-10 and have limited phagocytosis capacity. In this work, phagocytosis was investigated using $S$. mutans as a well-recognized representative of cariogenic bacteria. Based on our earlier investigations demonstrating that macrophage phagocytosis varies in function of the bacterial species $^{20}$, S. mutans was selected as an 


\section{HE}
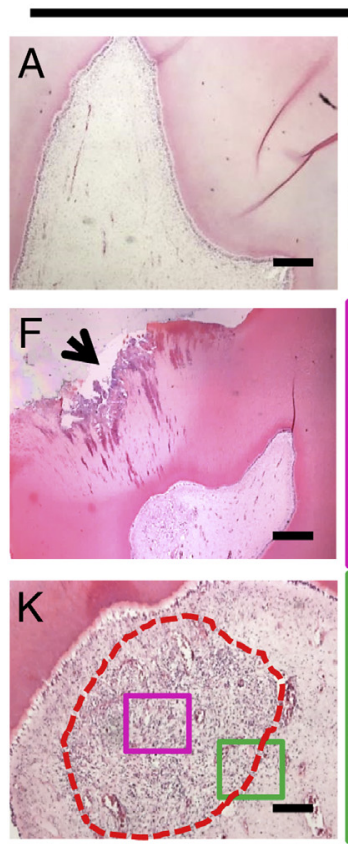
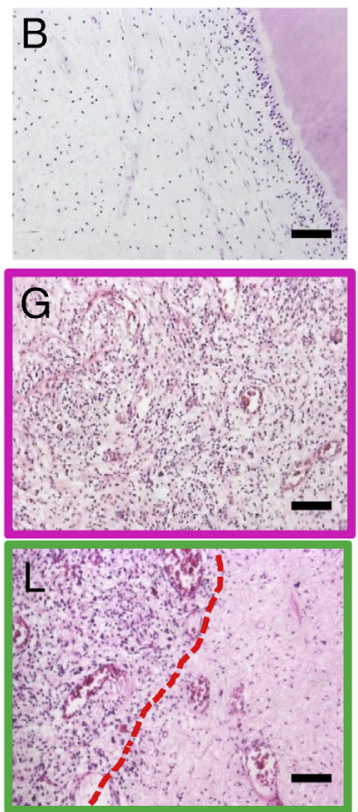

CD86 (M1)
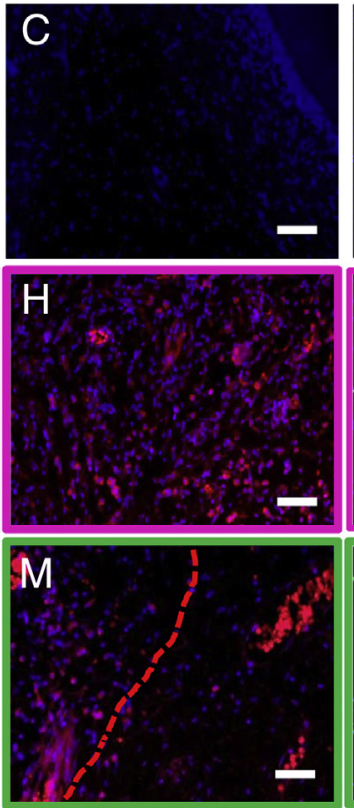

CD206 (M2)
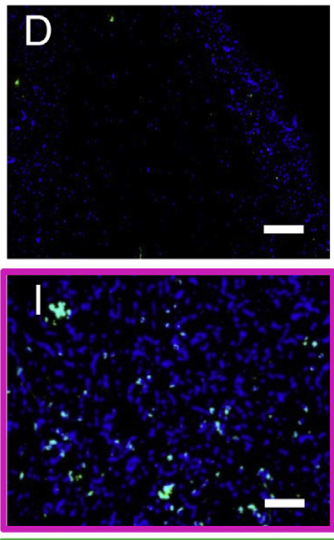

Control
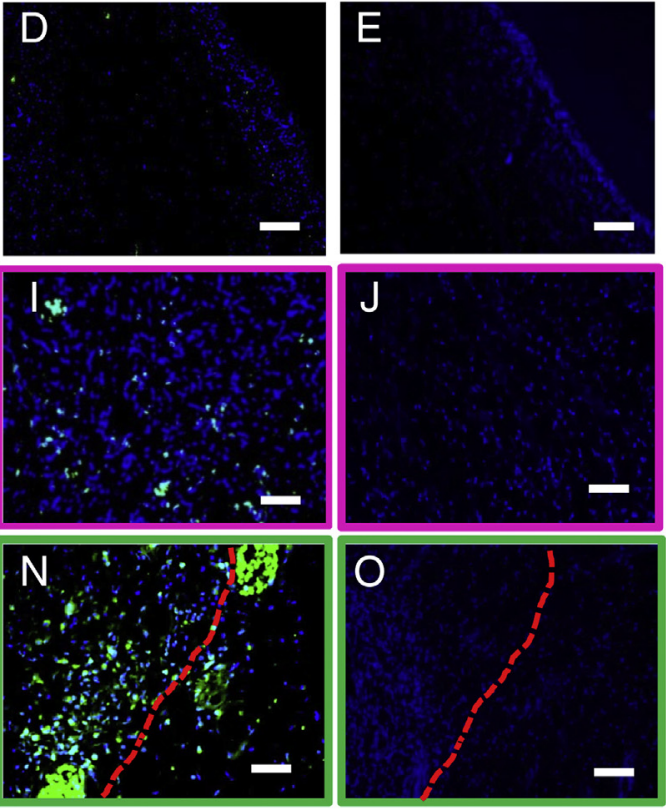

FIGURE 3 - M1 and M2 macrophage localization in human tooth sections. $(A-E)$ Sound and $(F-0)$ carious human tooth sections were stained with $(A, B, F, G, K$, and $L)$ H\&E. Immunofluorescence did not show any $(C)$ CD86 or $(D)$ CD206 and highlighted the absence of M1 and M2 macrophage in sound teeth. In carious tooth sections stained with H\&E, $(F)$ the black arrow indicates the carious lesion progression in the dentin and $(K)$ the red dots limit the resulting inflammatory reaction zone. (G-J) The magenta frame shows the central zone of the inflammatory area, and $(L-0)$ the green frame shows the inflammatory reaction peripheral zone. Immunostaining of CD86 (in red) indicates an intense labeling in the central inflammatory zone highlighting the presence of $(H)$ numerous M1 macrophages and $(M)$ much less in the peripheral zone. Immunostaining of CD206 (in green) shows an intense labeling at the peripheral zone indicating $(N) \mathrm{M} 2$ localization more in the peripheral than in $(I)$ the central area of the inflammatory reaction zone. $(E, J$, and $O)$ Isotypes controls were negative. Nuclei were counterstained with DAPI (blue). Scale bars: $A$ and $F=500 \mu \mathrm{m} ; K=200 \mu \mathrm{m}$; and $B, C, D, E, G, H, I, J, L, M, N$, and $O=50 \mu \mathrm{m}$

example to study the effect of fibroblast treatment conditions on the differentiated macrophage phagocytic capacity of the same strain.

Interestingly, distinct cytokine secretion profiles were obtained depending on the type of fibroblast stimulation. When MO macrophages were incubated with injured fibroblast supernatants (without LTA), they showed a high IL-10 secretion level and a moderate phagocytosis capacity. This profile is very similar to M2 macrophages. However, when they were incubated with supernatants of injured and LTA-stimulated fibroblasts, MO macrophages secreted a high level of TNF- $\alpha$ and a low level of IL-10 and had high phagocytic capacity. This profile is very similar to M1 macrophages. However, it should be noted that when MO macrophages were incubated with injured fibroblast supernatants, they secreted a low level of TNF- $\alpha$, whereas after incubation with injured and LTA-induced fibroblast supernatants, they secreted a low level of IL-10. This secretion level was very low compared with $\mathrm{M} 1$ and $\mathrm{M} 2$ reference macrophages. This indicates that we do not obtain a homogenous differentiation of cells into $\mathrm{M} 1$ or $\mathrm{M} 2$ but rather a balance of the 2 cell types with a predominant phenotype. These data demonstrate that injured and LTAstimulated fibroblasts, simulating both the presence of bacteria and cell damage, induce differentiation into $\mathrm{M} 1$, whereas the surrounding fibroblasts without a direct contact with bacteria induce macrophage differentiation into M2. The reason why fibroblasts enhance M2 polarization after injury without exposure to LTA is still unclear. Injured pulp cells have been shown to release DAMPs. DAMPs are not only danger signals but also central players in tissue inflammation and repair. Indeed, after sterile injury to pulp cells, recent data have demonstrated that DAMPs induced an NLRP3 inflammasome-dependent sterile inflammatory response that enhanced dental pulp cell migration, proliferation, and odontogenic differentiation ${ }^{25}$. It could be hypothesized that upon cariogenic bacteria invasion, 2 zones can be distinguished. The first zone that can be directly exposed to cariogenic bacteria is located beneath the carious dentin. It is simulated in vitro by injuring the cells physically and incubating them with LTA. The second zone is located at the periphery of this zone. This zone receives DAMPs secreted from injured/damaged cells without being subjected to bacteria directly. The absence of direct contact between fibroblasts and bacteria in this condition is favorable to M2 differentiation. The pulp fibroblast-released cytokines can mediate this differentiation as demonstrated in cardiac tissue. Indeed, upon LPS stimulation, the release of TNF- $\alpha$ and monocyte chemoattractant protein 1 by cardiac fibroblasts allow monocyte differentiation into M1, whereas IL-10 and IL- 5 secretion by the same cells allow M2 differentiation ${ }^{17}$.

Our data clearly demonstrate that pulp fibroblasts interact with macrophages and actively influence their differentiation and that this is highly dependent on the exposure of pulp fibroblasts to injury or bacterial infection. Thus, macrophage differentiation seems to be rather reversible and dynamic and can be modulated by the environment as demonstrated in cocultures of macrophages with Schwann cells, which induced their differentiation into $\mathrm{M} 2^{16}$.

During the inflammatory process, it has long been believed that macrophages are strictly of the M1 phenotype, whereas during the healing process they are strictly of the M2 phenotype. A previous study demonstrated 


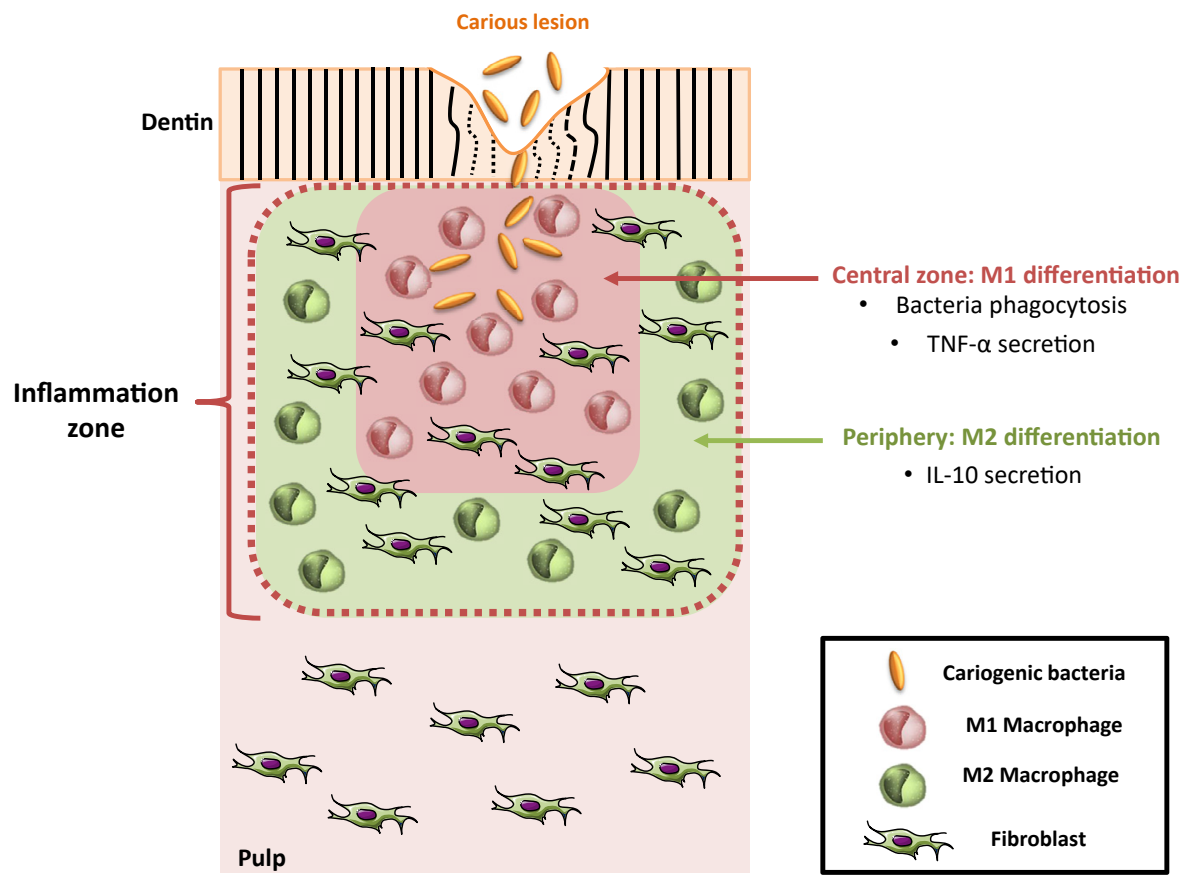

FIGURE 4 - A schematic representation of differentiated macrophage localization in the inflammatory zone under caries. During the carious process, cariogenic bacteria (in orange) invade the dental pulp through the dentin tubules. Pulp fibroblasts close to the carious front are in contact with both damaged cell signals released by odontoblasts and pulp cells and bacteria. To reflect this situation in vitro, pulp fibroblasts were stimulated by LTA and injured. The macrophages recruited to this area are exposed to fibroblast secretion and differentiate into M1 macrophages involved in cariogenic bacteria phagocytosis, as demonstrated in the gentamycin assay, and proinflammatory TNF- $\alpha$ secretion. More distant fibroblasts, which are not subjected to bacterial invasion directly, differentiate into M2 macrophages involved in LL-10 anti-inflammatory cytokine secretion, limiting the immune response and preventing the underlying tissue damage. M1 macrophages allow the control and elimination of bacteria, whereas M2 macrophages at the periphery of the inflammatory zone dampen the inflammation reaction and limit pulp necrosis.

the presence of $\mathrm{M} 1$ in the inflammation zone of teeth with deep caries, whereas M2 macrophages were detected in teeth with superficial caries, under the carious dentin, as well as after pulp capping with mineral trioxide aggregate along the nerve fibers in the wounded tissue ${ }^{16}$. A novel aspect of our study is the demonstration that both $\mathrm{M} 1$ and $\mathrm{M} 2$ are present in the inflammation area of carious teeth; M1 cells were predominant in the center of the inflammatory zone. They occupy the area beneath the invading cariogenic bacteria and represent the first macrophage defense line in the pulp, whereas M2 mainly occupied the peripheral zone. These cells have an anti-inflammatory activity, which limits tissue damage during the inflammation. However, it should be noted that a mixed M1/M2 population is present in both zones. The presence of both phenotypes in central and peripheral zones is important to regulate the balance of pulp inflammation and repair. The phenotype plasticity is essential during the inflammation process to avoid significant cell damage and chronic inflammation ${ }^{34,35}$. Our results reporting the presence of a mixed $\mathrm{M} 1 / \mathrm{M} 2$ population are in line with these data.

Overall, taking into consideration both macrophage differentiation in vitro and their localization in vivo allows us to advance the following hypothesis on fibroblast interaction with macrophages: during the carious process, the pulp fibroblasts subjected to cariogenic bacteria and sensing cell damage signals stimulate M1 macrophage differentiation. These cells have a high phagocytic capacity and lead to bacterial elimination by phagocytosis. The surrounding fibroblasts that are not in direct contact with bacteria induce M2 differentiation with antiinflammatory activity, limiting pulp tissue damage (Fig. 4). It can be assumed that this M1/M2 balance allows pathogen elimination and provides a control of pulpal inflammation that is required for initiating the healing process.

\section{ACKNOWLEDGMENTS}

Supported by Aix-Marseille University and CNRS. The authors deny any conflicts of interest related to this study.

\section{REFERENCES}

1. Farges JC, Alliot-Licht B, Renard E, et al. Dental pulp defence and repair mechanisms in dental caries. Mediators Inflamm 2015;2015:1-16.

2. Giraud T, Jeanneau C, Bergmann M, et al. Tricalcium silicate capping materials modulate pulp healing and inflammatory activity in vitro. J Endod 2018;44:1686-91. 
3. Yumoto H, Hirao K, Hosokawa Y, et al. The roles of odontoblasts in dental pulp innate immunity. Jpn Dent Sci Rev 2018;54:105-17.

4. Farges JC, Keller JF, Carrouel F, et al. Odontoblasts in the dental pulp immune response. J Exp Zool B Mol Dev Evol 2009;312B:425-36.

5. Giraud T, Rufas P, Chmilewsky F, et al. Complement activation by pulp capping materials plays a significant role in both inflammatory and pulp stem cells' recruitment. J Endod 2017;43:1104-10.

6. Nakakura-Ohshima K. Possible role of immunocompetent cells and the expression of heat shock protein-25 in the process of pulpal regeneration after tooth injury in rat molars. J Electron Microsc (Tokyo) 2003;52:581-91.

7. Zhang J. The existence of CD11c+ sentinel and F4/80 + interstitial dendritic cells in dental pulp and their dynamics and functional properties. Int Immunol 2006;18:1375-84.

8. Martinez FO. Macrophage activation and polarization. Front Biosci 2008;13:453.

9. Mantovani A, Sica A, Sozzani S, et al. The chemokine system in diverse forms of macrophage activation and polarization. Trends Immunol 2004;25:677-86.

10. Novak ML, Koh TJ. Macrophage phenotypes during tissue repair. J Leukoc Biol 2013;93:87581.

11. Nakanishi T, Takahashi K, Hosokawa Y, et al. Expression of macrophage inflammatory protein 3alpha in human inflamed dental pulp tissue. J Endod 2005;31:84-7.

12. Adachi T, Nakanishi T, Yumoto H, et al. Caries-related bacteria and cytokines induce CXCL10 in dental pulp. J Dent Res 2007;86:1217-22.

13. Cooper PR, Takahashi Y, Graham LW, et al. Inflammation-regeneration interplay in the dentinepulp complex. J Dent 2010;38:687-97.

14. Park HC, Quan H, Zhu T, et al. The effects of M1 and M2 macrophages on odontogenic differentiation of human dental pulp cells. J Endod 2017;43:596-601.

15. Takei E, Shigetani $Y$, Yoshiba K, et al. Initial transient accumulation of M2 macrophageassociated molecule-expressing cells after pulpotomy with mineral trioxide aggregate in rat molars. J Endod 2014;40:1983-8.

16. Yoshiba N, Edanami N, Ohkura N, et al. M2 phenotype macrophages colocalize with Schwann cells in human dental pulp. J Dent Res 2020;99:329-38.

17. Humeres C, Vivar R, Boza P, et al. Cardiac fibroblast cytokine profiles induced by proinflammatory or profibrotic stimuli promote monocyte recruitment and modulate macrophage M1/M2 balance in vitro. J Mol Cell Cardiol 2016;101:69-80.

18. Chmilewsky F, Jeanneau C, Laurent P, About I. Pulp fibroblasts synthesize functional complement proteins involved in initiating dentin-pulp regeneration. Am J Pathol 2014;184:19912000.

19. Coil J, Tam E, Waterfield JD. Proinflammatory cytokine profiles in pulp fibroblasts stimulated with lipopolysaccharide and methyl mercaptan. J Endod 2004;30:88-91.

20. Le Fournis C, Hadjichristou C, Jeanneau C, About I. Human pulp fibroblast implication in phagocytosis via complement activation. J Endod 2019;45:584-90.

21. Tran-Hung L, Mathieu S, About I. Role of human pulp fibroblasts in angiogenesis. J Dent Res 2006;85:819-23.

22. Chmilewsky F, About I, Chung SH. Pulp fibroblasts control nerve regeneration through complement activation. J Dent Res 2016;95:913-22.

23. Jeanneau C, Lundy FT, EI Karim IA, About I. Potential therapeutic strategy of targeting pulp fibroblasts in dentin-pulp regeneration. J Endod 2017;43:S17-24.

24. Cooper PR, Smith AJ. Molecular mediators of pulp inflammation and regeneration. Endod Topics 2013;28:90-105.

25. Al Natour B, Lundy FT, Moynah PN, et al. Odontoblast cell death induces NLRP3 inflammasomedependent sterile inflammation and regulates dental pulp cell migration, proliferation and differentiation. Int Endod J 2021;54:941-50.

26. Rufas $P$, Jeanneau C, Rombouts $C$, et al. Complement C3a mobilizes dental pulp stem cells and specifically guides pulp fibroblast recruitment. J Endod 2016;42:1377-84.

27. Tsuchiya S, Yamabe M, Yamaguchi Y, et al. Establishment and characterization of a human acute monocytic leukemia cell line (THP-1). Int J Cancer 1980;26:171-6. 
28. Murray PJ, Allen JE, Biswas S, et al. Macrophage activation and polarization: nomenclature and experimental guidelines. Immunity 2014;41:14-20.

29. Laroux FS, Romero X, Wetzler L, et al. Cutting edge: MyD88 controls phagocyte NADPH oxidase function and killing of gram-negative bacteria. J Immunol 2005;175:5596-600.

30. Téclès $\mathrm{O}$, Laurent $\mathrm{P}$, Zygouritsas $\mathrm{S}$, et al. Activation of human dental pulp progenitor/stem cells in response to odontoblast injury. Arch Oral Biol 2005;50:103-8.

31. Tan HY, Wang N, Man K, et al. Autophagy-induced RelB/p52 activation mediates tumourassociated macrophage repolarisation and suppression of hepatocellular carcinoma by natural compound baicalin. Cell Death Dis 2015;6:e1942.

32. Chávez-Galán L, Ocaña-Guzmán R, Torre-Bouscoulet L, et al. Exposure of monocytes to lipoarabinomannan promotes their differentiation into functionally and phenotypically immature macrophages. J Immunol Res 2015;2015:984973.

33. Neves VC, Yianni V, Sharpe PT. Macrophage modulation of dental pulp stem cell activity during tertiary dentinogenesis. Sci Rep 2020;10:20216.

34. Porcheray F, Viaud S, Rimaniol AC, et al. Macrophage activation switching: an asset for the resolution of inflammation. Clin Exp Immunol 2005;142:481-9.

35. Benoit M, Desnues B, Mege JL. Macrophage polarization in bacterial infections. J Immunol 2008;181:3733-9. 